\title{
INTEGRATION OF A FILM BULK ACOUSTIC RESONATOR WITH MICROFLUIDIC CHANNELS FOR BIOMEDICAL SENSING IN LIQUID
}

\author{
W. Xu, X. Zhang, H. Yu, A. Abbaspour-Tamijani and J. Chae \\ Department of Electrical Engineering, Arizona State University, Tempe, Arizona, USA
}

\begin{abstract}
This paper reports an integration of microfluidic channels with a film bulk acoustic resonator (FBAR) for in liquid biomedical sensing applications. The device consists of a zinc oxide $(\mathrm{ZnO})$ film based longitudinal mode FBAR and parylene encapsulated microfluidic channels. By confining the liquid with the height comparable to the acoustic wavelength of the FBAR in the microfluidic channels, the quality factor $(Q)$ of the resonating system significantly increases, resulting in very high sensitivity FBAR sensor for liquid samples. We demonstrate a FBAR with $Q$ up to 120 , an improvement of more than 8 times over that of existing FBARs in liquid. A bio-molecular (streptavidine) sensing test presents a sensitivity of $638 \mathrm{~Hz} \mathrm{~cm} / \mathrm{ng}$ and an extremely fine resolution of $2.025 \mathrm{ng} / \mathrm{cm}^{2}$ of the sensor.
\end{abstract}

\section{INTRODUCTION}

Compared to fluorescent and radioactive labeling methods, label-free bimolecular sensors in liquid media are useful for various applications ranging from pharmaceutical research to medical diagnostics, partially due to the fact that label-free detection eliminates labeling process which may induce modification of chemical properties of target molecules [1]. However, widely used label-free protein detection techniques, such as surface plasmon resonance (SPR) [2, 3] and quartz crystal microbalances (QCM) [4, 5], are significantly less sensitive than labeling methods $[1,6]$. In recent years, FBAR has shown great potentials of being high sensitivity biosensors. State-of-the-art ZnO-based FBAR biosensors reported in recent literature exhibit a mass sensitivity of up to $1000 \mathrm{~Hz} \mathrm{~cm} / \mathrm{ng}$ in air, over 1000 times higher than that of traditional QCMs [7-11]. Similar to QCMs, FBAR consists of a piezoelectric layer of 1 2 $\mu \mathrm{m}$ sandwiched between two metal electrodes, with an operating frequency above 1 GHz. $Q$ of 500 at $1 \mathrm{GHz}$ can be easily achieved in the air or vacuum, which produces extremely high detecting resolutions. However, in the liquid environments $Q$ significantly reduces due to the viscous damping of the contacting liquid. For instance in water $Q$ usually drops by a factor of $10-100$, which reduces the sensing ability of the sensor. As $Q$ decreases the minimum detectable frequency shift increases, thus the sensible mass resolution reduces roughly by the same factor. Although shear mode vibration instead of longitudinal mode can reduce the $Q$ degradation, it unavoidably lowers sensitivity [12, 13]. In shear mode, $Q$ has been reported up to 100-150 in water; however the sensitivity drops approximately to $1 / 3$ of the longitudinal mode resonators of similar dimensions. Clearly, a high $Q$ resonator in liquid with uncompromised sensitivity is the key to achieve a high resolution, high sensitivity biosensor.

\section{APPROACH AND MODELING}

A typical FBAR have a thickness of $1-2 \mu$ m piezoelectric thin film such as $\mathrm{ZnO}$ or AlN mechanically supported by a dielectric layer, which has its fundamental resonance frequency above $1 \mathrm{GHz}$ in longitudinal mode. FBARs usually have high Qs from several hundreds up to a couple of thousands in the air, because the acoustic energy is well entrapped in the resonator body due to very large impedance mismatch between solid materials and the air.
The $Q$ drops significantly (12-25) in water since acoustic impedance mismatch between liquid and solid is much smaller than that between solid and air. Therefore the acoustic waves generated inside of the solid resonator partially transmit into the liquid and dissipate, consequently reduce $Q$.

The approach that we present is to form a very thin liquid layer on the solid resonator so that the acoustic wave length inside the liquid is comparable to the thickness of the thin liquid layer. This allows minimizing the energy dissipation inside the liquid. In order to form the thin liquid layer, we use microfluidic channels integrated with FBAR. Figure 1 illustrates the schematic design of the FBAR sensor integrated with microfluidic channels. A threelayer FBAR $(\mathrm{Al} / \mathrm{ZnO} / \mathrm{Au})$ is fabricated on a suspended silicon nitride (SiN) membrane. A parylene enclosure built on top of the FBAR forms the microfluidic channels. The channel height is precisely defined by a sacrificial layer, and inlet / outlet ports are accessible from the backside of the device.

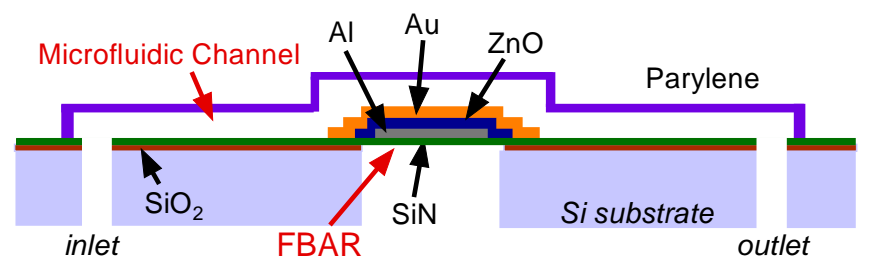

Figure 1: Schematic structure of an FBAR sensor integrated with the microfluidic channel.

We use a transmission-line model (Figure 2) for the multilayer composite resonator to estimate its $Q$ behavior. The model consists of six physical layers of the device: SiN supporting membrane, Al electrode, piezoelectric $\mathrm{ZnO}$ layer, $\mathrm{Au}$ electrode, water layer confined in the microfluidic channel, and parylene microfluidic channel. Since the four solid layers have negligible loss compared to the liquid or polymer, we simplify the model that attenuation occurs only in liquid and polymer.

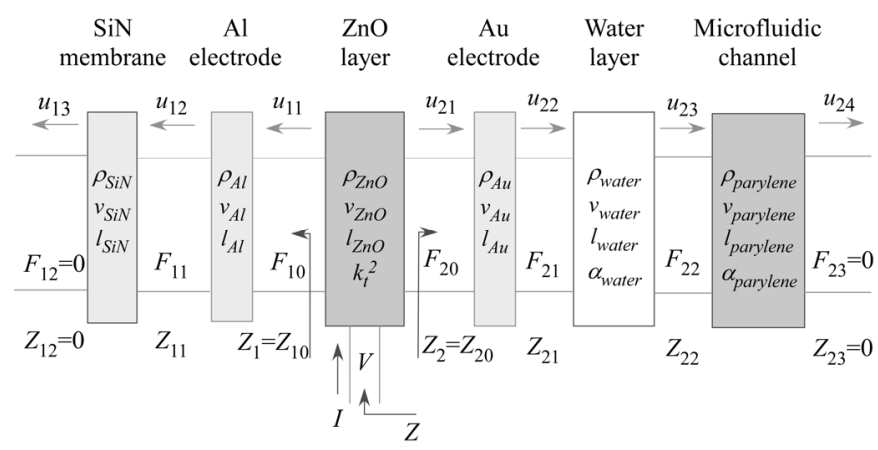

Figure 2: Equivalent transmission-line model of the FBAR with multilayer composites.

The piezoelectric $\mathrm{ZnO}$ layer is modeled as a three port component which has an electrical terminal pair (voltage $V$ and 
current $I$ ), the back and front acoustic ports denoted as force $F$ and displacement $u$. From the equivalent circuit model of Mason's inline model of a piezoelectric film, the electric input impedance of the system can be written as [14]:

$$
Z=\frac{1}{j \omega C_{0}}\left[1-\frac{k_{t}^{2}}{\gamma} \cdot \frac{\left(z_{1}+z_{2}\right) \cdot \sin \gamma+j \cdot 2(1-\cos \gamma)}{\left(z_{1}+z_{2}\right) \cdot \cos \gamma+j \cdot\left(1+z_{1} z_{2}\right) \cdot \sin \gamma}\right]
$$

where $C_{0}=\varepsilon_{33} \cdot S / l_{Z n O}$ is the static capacitance of the resonator, $S$ is the effective area of the FBAR, $\varepsilon_{33}$ and $l_{Z n O}$ are the permittivity and thickness of the $\mathrm{ZnO}$ layer, $k_{t}^{2}$ is the electromechanical coupling coefficient of $\mathrm{ZnO}, \gamma=\omega l_{\mathrm{ZnO}} / v_{\mathrm{ZnO}}$ is the phase delay of the longitudinal acoustic wave with velocity $v_{\mathrm{ZnO}}$ in $\mathrm{ZnO}, \omega$ is the angular frequency, $z_{1}=Z_{1} / Z_{0}, z_{2}=Z_{2} / Z_{0}$ are the normalized acoustic loading impedance on both sides of the $\mathrm{ZnO}$ film, $Z_{0}=S \rho_{\mathrm{ZnO}} v_{\mathrm{ZnO}}$ is the acoustic impedance of the $\mathrm{ZnO}$ layer, and $\rho_{\mathrm{ZnO}}$ is the density, respectively.

$Z_{1}$ and $Z_{2}$ are cascaded from the adjacent component and loading impedance by (Figure 2):

$$
Z_{m n}=Z_{0 m n} \frac{Z_{m, n+1}+Z_{0 m n} \tanh \gamma_{0 m n} l_{0 m n}}{Z_{0 m n}+Z_{m, n+1} \tanh \gamma_{0 i j} l_{0 m n}}
$$

where $m=1$ and 2, indicating the impedance series on the left and right of $\mathrm{ZnO}, Z_{m n}$ is the loading impedance at the cross section after the $n^{\text {th }}$ component either on the right or left of the $\mathrm{ZnO}, Z_{0 m n}$, $l_{0 m n}$ are the acoustic impedance, thickness of the $j^{\text {th }}$ component, respectively, $\gamma_{0 \mathrm{mn}}=\alpha_{0 \mathrm{mn}}+j \beta_{0 \mathrm{mn}}, \alpha_{0 \mathrm{mn}}$ is the attenuation coefficient and $\beta_{0 \mathrm{mn}}$ is the angular wave number of the corresponding component. We define a boundary condition of $Z_{12}=Z_{23}=0$, stating the mechanically free at the parylene/air or SiN/air interfaces. We use MATLAB to calculate $Q$ :

$$
Q=\frac{f}{2} \frac{\partial \varphi(Z)}{\partial f}
$$

where $\varphi(Z)$ is the phase of the impedance $Z$ in eq. (1).

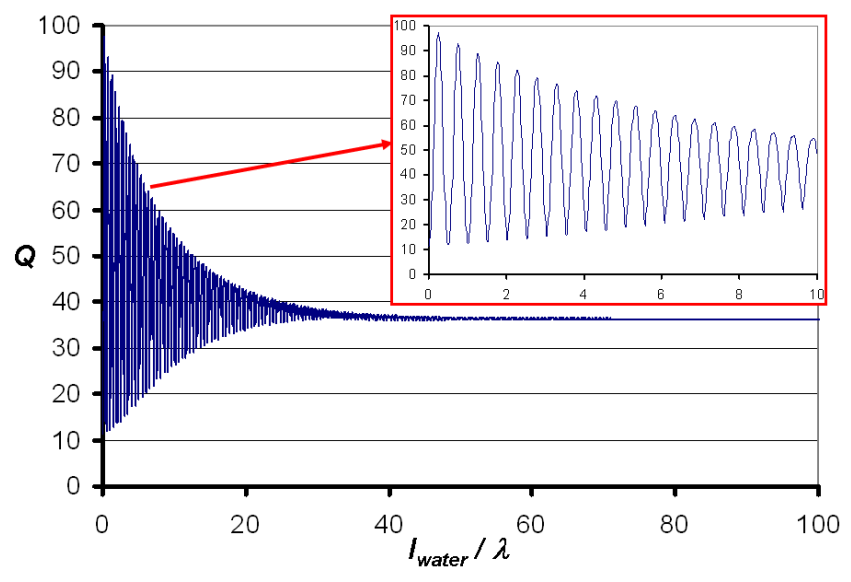

Figure 3: Theoretically predicted $Q$ versus the ratio of fluidic channel height to the acoustic wavelength in water.

Figure 3 shows the MATLAB simulation of a $0.5 \mu \mathrm{m}$ thick $\mathrm{ZnO}$ FBAR. We plot $Q$ in terms of the ratio of the thickness of water layer $\left(l_{\text {water }}\right)$ to acoustic wavelength in the water $(\lambda)$. The
FBAR has a resonance frequency of $2.0 \mathrm{GHz}$. As the ratio becomes large, above 30 , the $Q$ converges to low $(<40)$. In other words, the large portion of the acoustic energy generated inside the FBAR dissipates in the water if the thickness of the water layer is more than 30 times of the acoustic wavelength in the water. This region is where typical FBAR operates at in liquid. However, when the ratio is less than $30, Q$ starts oscillating. This is because the acoustic waves reflected from $\mathrm{ZnO} /$ /iquid and liquid/parylene boundaries alternatively interfere with each other constructively (in phase) or destructively (out of phase). If technologies allow implementing precisely ratioed structures of $\mathrm{ZnO} /$ liquid, it is possible to obtain high $Q$ at contacting liquid.

\section{EXPERIMENTS AND EVALUATION METHODS Fabrication}

Figure 4 illustrates the fabrication process flow. The FBAR sensor is fabricated using standard silicon micromachining technologies. Fabrication starts with a $<100>$ silicon wafer; $2000 \AA$ thick oxide is first thermally grown on the wafer, followed by a $6000 \AA$ thick low-stress LPCVD SiN layer as a mechanical supporting membrane. $1600 \AA$ thick $\mathrm{Al}$ is evaporated and patterned on the SiN layer as the bottom electrode. The $\mathrm{Al}$ electrode determines the effective area of the FBAR. $\mathrm{ZnO}$ is then sputtered for $0.55,2.1,4.2 \mu \mathrm{m}$. $300 \AA / 1500 \AA$ thick $\mathrm{Cr} / \mathrm{Au}$ is sputtered and lift-off patterned on the $\mathrm{ZnO}$ layer as a top electrode. We use Au as the top electrode due to its excellent conductivity and good affinity to biomolecular binding [15]. 3 $3 \mathrm{~m}$ thick photoresist is coated and patterned as a sacrificial layer on $\mathrm{Au}$, followed by parylene $\mathrm{C}$ film coating to form the fluidic channel enclosures. The parylene $C$ thin film is $3 \mu \mathrm{m}$ thick which provides enough mechanical strength.

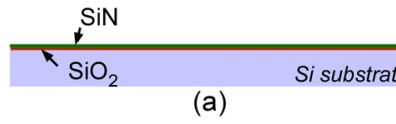

Al bottom electrode

(b)

$\mathrm{ZnO}$ piezoelectric film

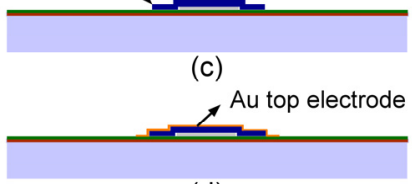

(d)

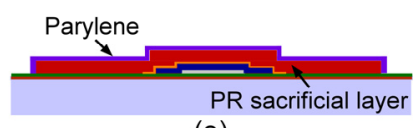

(e)

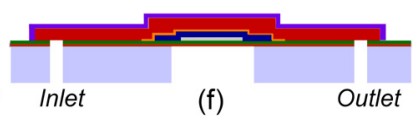

(f)

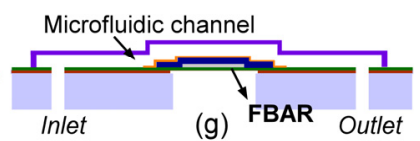

Figure 4: Fabrication process of a FBAR integrated with a microfluidic channel: a) thermally grown $\mathrm{SiO}_{2}$ and low stress $\mathrm{SiN}$ by LPCVD on Si substrate; b) Al deposition and patterning; c) $\mathrm{ZnO}$ deposition by sputtering and patterning; d) deposition of $\mathrm{Cr} / \mathrm{Au}$ by patterning; e) photoresist patterns as sacrificial layer and coating of parylene $C$; f) Deep RIE to form inlet/outlet of channels and release the membrane; g) removing $\mathrm{SiO}_{2}$ beneath $\mathrm{SiN}$ and sacrificial PR; supercritical $\mathrm{CO}_{2}$ drying to release the microfluidic channel.

The wafer is etched through from the backside by Deep RIE (Reactive Ion Etch) to release the SiN membranes and to form the channel inlets/outlets. Finally, the sacrificial photoresist layer is removed by soaking devices in acetone for overnight and the microfluidic channels are dried by supercritical $\mathrm{CO}_{2}$ process. Additionally, the $\mathrm{SiO}_{2}$ layer underneath $\mathrm{SiN}$ which works as a stopping layer for Deep RIE is removed by buffered oxide etchant to achieve a smooth $\mathrm{SiN}$ supporting membrane. The $\mathrm{SiO}_{2}$ stopping 
layer may not be very smooth due the physical bombardment during Deep RIE process. The fabricated FBAR integrated with the microfluidic channel is shown in Figure 5.

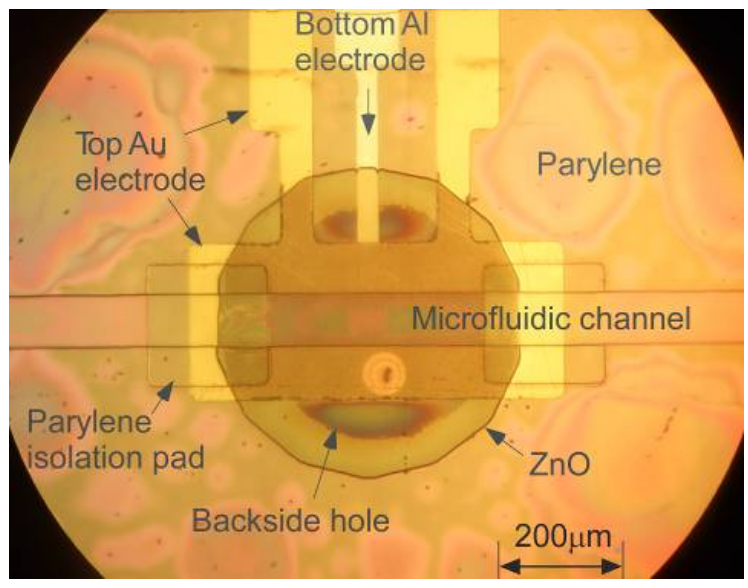

Figure 5: Top view of the fabricated FBAR integrated with the microfluidic channel.

\section{$Q$ Measurements}

$Q$ of the fabricated devices are measured for three different cases; in the air, drenched in the water and water in the microfluidic channel. These correspond to no damping, full damping and partial damping, respectively.

Chips are mounted on a probe station and characterized using HP 8510C network analyzer, 85105A millimeter-wave controller and $8517 \mathrm{~B}$ S-Parameter test set. The test setup is shown in Figure 6. We record $S_{11}$ and the impedance $Z$ of the sensor is extracted from the $S_{11}$ spectrum. Then, the $Q$ of resonators is calculated by eq. (3).

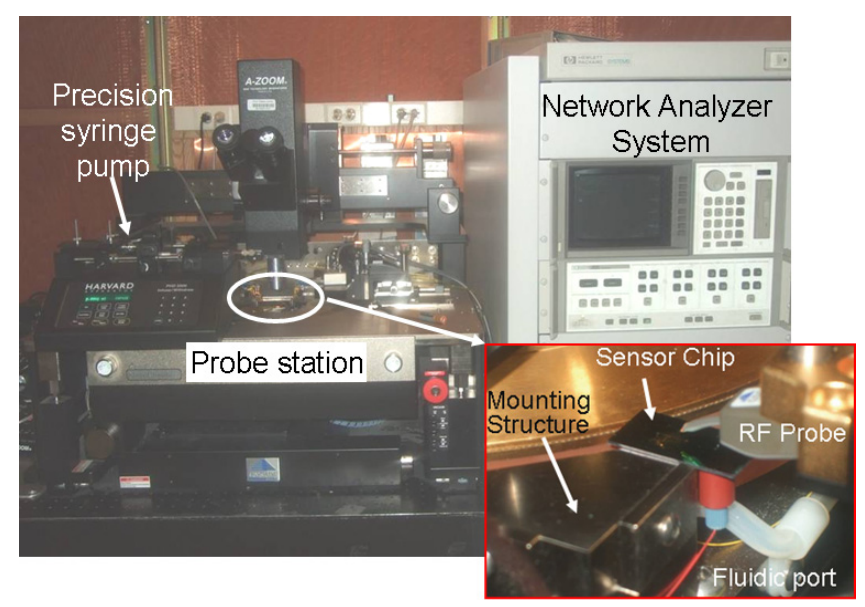

Figure 6: Test setups; testing device on a probe station, connected with a syringe pump and a network analyzer system.

$2.1 \mu \mathrm{m}$ thick ZnO FBAR has a parallel resonant frequency of 1.05GHz. In the air, $Q$ factor reaches $\sim 250$. We fill up the backside hole with water to measure the $Q$ at full damping. The water is practically infinitely deep since the depth is more than 300 $\mu \mathrm{m}$, much larger than the acoustic wavelength in water $(\sim 1.42 \mu \mathrm{m})$. $Q$ drops approximately to 7-15, which matches well with the previous reports $[13,16]$. Now, we flow water through the microfluidic channel to measure $Q$ at partial damping. Water flows at a constant rate $(25 \mu \mathrm{L} / \mathrm{hr})$ in the microfluidic channel driven by a precisely controlled infusion syringe pump. The $Q$ drops from 250 to 120 when the water reaches the Au surface of the FBAR and remains nearly constant afterward. Compared to the reported longitudinal FBARs in water, our devices show 8 to 10 times higher $Q$, high enough to compete with the shear-mode FBARs.

Table 1 lists the measured $Q$, corresponding channel heights and the heights to wavelength ratios. Note that $Q$ of partial damping varies widely. We believe this is due to the variation of water heights. As the water heights change $Q$ changes significantly in the partial damping regime as shown in Figure 3. Figure 7 compares the measured impedance spectrum of no damping (in the air), full damping (drenched in water), and partial damping (water in microfluidic channels).

Table 1: Measured Q for different damping conditions.

\begin{tabular}{|c|c|c|c|c|}
\hline \multicolumn{2}{|c|}{$\mathrm{ZnO}$ thickness $(\mu \mathrm{m})$} & 0.55 & 2.1 & 4.1 \\
\hline \multicolumn{2}{|c|}{ Resonant freq. (GHz) } & 2.2 & 1.05 & 0.6 \\
\hline \multirow{3}{*}{ No damping } & $l_{\text {water }}(\mu \mathrm{m})$ & -- & -- & -- \\
\hline & $l_{\text {water }} / \lambda$ & -- & -- & -- \\
\hline & $Q$ & 220 & 240 & 430 \\
\hline \multirow{3}{*}{ Full damping } & $l_{\text {water }}(\mu \mathrm{m})$ & 500 & 500 & 500 \\
\hline & $l_{\text {water }} / \lambda$ & -- & -- & -- \\
\hline & $Q$ & 12 & 7 & 15 \\
\hline \multirow{3}{*}{ Partial damping } & $l_{\text {water }}(\mu \mathrm{m})$ & 3.0 & 3.0 & 3.0 \\
\hline & $l_{\text {water }} / \lambda$ & 4.45 & 2.12 & 1.21 \\
\hline & $Q$ & $35 \sim 40$ & $65 \sim 120$ & $85 \sim 110$ \\
\hline
\end{tabular}

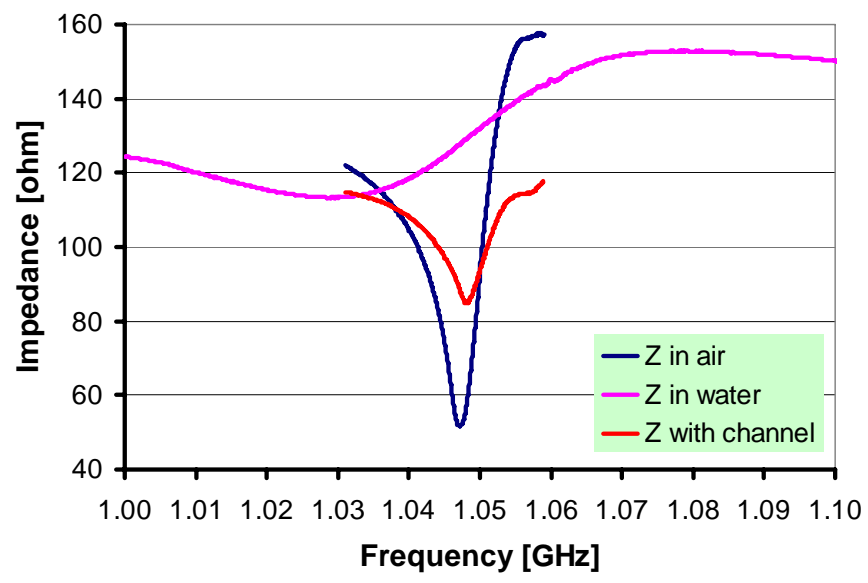

Figure 7: Impedance spectrums of FBAR in the air (no damping), in water (full damping), and in the microfluidic channel (partial damping).

\section{Bio-sensing testing}

Streptavidine is a protein which strongly binds to Au. As a proof-of-concept bio-sensing test, we use streptavidine molecules to characterize the fabricated FBAR sensor. The top electrode is made of $\mathrm{Au}$, which forms a permanent bond with streptavidine flowing through the microfluidic channels.

Figure 8 shows the test sequence. Phosphate Buffer Solution (PBS) first flows through the microfluidic channel, at a constant 
rate of $25 \mu \mathrm{L} / \mathrm{hr}$. After the flow stabilizes, the PBS is replaced by $0.1 \mu \mathrm{M}$ streptavidine solution. A frequency shift occurs immediately. This corresponds to the density and viscosity difference between these two solutions. After the streptavidine binding saturated the gold surface, we switch back to PBS. A permanent frequency shift of $90 \mathrm{kHz}(86 \mathrm{ppm})$ is measured. The frequency shift indicates the mass loading by permanent streptavidine bound to the Au surface.

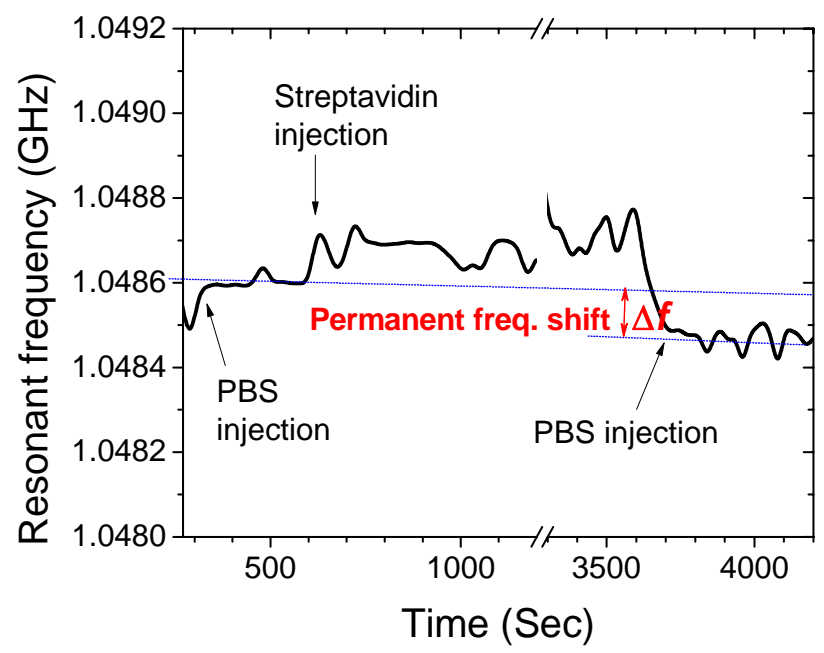

Figure 8: Measured parallel resonance frequency shift $(\Delta f)$ of the FBAR sensor over time ( $t$ ) due to the mass loading on gold electrodes resulted by steptavidine-gold bindings.

For the proof-of-concept test, the resonator has a $Q$ of 80 in both PBS and streptavidine solutions. The sensitivity is calculated as $638 \mathrm{~Hz} \mathrm{~cm} / \mathrm{ng}$, which indicates a resolution of $2.025 \mathrm{ng} / \mathrm{cm}^{2}$, approximately 10 times better than reported resolution.

\section{CONCLUSION}

We significantly improve $Q$ of FBAR in liquid environments by confining the liquid in a micron range to minimize the acoustic energy dissipation. We demonstrate the $Q$ of FBAR reaches 120, which is at least 8 times higher than reported $Q$ in liquid environments. The $Q$ improvement enhances the sensitivity of the FBAR sensor, thus increases the resolution of the sensor. Future efforts include optimizing the microfluidic channel to achieve higher $Q$ and demonstrate high sensitivity biosensors for various biomolecules.

\section{REFERENCES}

1. T. Burg, and S. Manalis, "Suspended microchannel resonators for biomolecular detection", Appl. Phys. Lett., 83, 2698, (2003).

2. J. Homola,, "Present and future of surface plasmon resonance biosensors", Analytical and Bioanalytical Chemistry, 377, 528 (2003).

3. J. Homola, S. Yee, and G. Gauglitz, "Surface plasmon resonance sensors: review", Sensors and Actuators B, Chemical,. 54, 3, (1999).

4. C. K. O'Sullivan, and G. Guilbault, "Commercial quartz crystal microbalances-theory and applications", Biosensors and Bioelectronics,. 14, 663, (1999).

5. M. Jonsson, et al., "Quartz crystal microbalance biosensor design", Sensors and Actuators B (Chemical), 123, 21, (2007).

6. T. Burg, et al., "Vacuum-packaged suspended microchannel resonant mass sensor for biomolecular detection", Journal of Microelectromechanical Systems, 15, 1466, (2006).

7. J. Bender and J. Krim, "Applications of the Piezoelectric Quartz Crystal Microbalance for Microdevice Development", Microscale Diagnostic Techniques. 227, (2005).

8. R. Gabl, et al., "First results on label-free detection of DNA and protein molecules using a novel integrated sensor technology based on gravimetric detection principles", Biosens. Bioelectron., 19, 615, (2004).

9. L. Yan, et al., "Piezoelectrically transduced low-impedance microelectromechanical resonators", Appl. Phys. Lett., 87, 154103, (2005).

10. S. Lee, K.H. Yoon, and J. Lee, "Influence of electrode configurations on the quality factor and piezoelectric coupling constant of solidly mounted bulk acoustic wave resonators", J. Appl. Phys., 92, 4062, (2002).

11. Z. Yan, et al., "ZnO-based film bulk acoustic resonator for high sensitivity biosensor applications", Appl. Phys. Lett., 90, 143503, (2007).

12. G. Wingqvist, et al., "Shear mode AlN thin film electroacoustic resonant sensor operation in viscous media", Sens. Actuators B, Chem., 123, 466, (2007).

13. J. Weber, et al., "Shear mode FBARs as highly sensitive liquid biosensors". Sens. Actuators A, Phys., 128, 84, (2006).

14. Y. Zhang, Z. Wang, and J. D. N. Cheeke, "Resonant spectrum method to characterize piezoelectric films in composite resonators", IEEE Trans. Ultrason. Ferroelectr. Freq. Control, 50, 321 (2003).

15. E. Ostuni, L. Yan, and G.M. Whitesides, "The interaction of proteins and cells with self-assembled monolayers of alkanethiolates on gold and silver", Colloids and Surfaces B: Biointerfaces, 15, 3, (1999).

16. H. Zhang, et al., "A film bulk acoustic resonator in liquid environments”, J. Micromech. Microeng., 15, 1911, (2005). 